\title{
Assessment of Clinical Pharmacy Interventions to Reduce Outpatient Use of High-Risk Medications in the Elderly
}

\author{
Sarah C. Weddle, PharmD, BCPS; A. Shaun Rowe, PharmD, BCPS; Julie W. Jeter, MD; \\ Rachel C. Renwick, PharmD, BCPS; Shaunta' M. Chamberlin, PharmD, BCPS, FCCP; \\ and Andrea S. Franks, PharmD, BCPS
}

\begin{abstract}
BACKGROUND: Use of high-risk medications in the elderly (HRME) and drug-disease (Rx-DIS) interactions in the elderly, as defined by the Healthcare Effectiveness Data and Information Set (HEDIS) Measures, are significantly associated with mortality, hospital admission, and need for emergency care. No published studies to date evaluate interventions to reduce the use of HEDIS-defined HRME, although many studies have postulated a beneficial effect of such interventions.
\end{abstract}

OBJECTIVE: To evaluate the effect of pharmacist interventions on use of HRME and Rx-DIS interactions in the outpatient elderly population.

METHODS: This retrospective cohort study was conducted in a residentbased family medicine clinic. Patients aged $\geq 65$ years were prospectively screened for the use of HRME and Rx-DIS interactions before their visits with their primary care providers. If HRME or Rx-DIS interactions were noted, the clinical pharmacist sent messages to the physicians through the electronic medical record, alerting them of the findings with suggestions of safer alternative agents, if applicable. The recommendation acceptance rate was assessed and then compared with a historical control from a similar time frame. The primary outcome was assessed with a chi square analysis. Secondary outcomes were assessed with descriptive statistics, chi square test, and Fisher's exact test.

RESULTS: HRME and/or Rx-DIS interactions were changed $25.9 \%$ of the time in the pharmacist intervention group compared with only $2.0 \%$ of the time in the historical control group $(P=0.001)$. The most frequently changed medication classes included skeletal muscle relaxants, benzodiazepines, and nonsteroidal anti-inflammatory drugs. 0ver $85 \%$ of the medication changes were preserved at the end of the study period. There was no difference between groups in the number of patients with HRME or Rx-DIS interactions.

CONCLUSIONS: Clinical pharmacy interventions result in significant reductions in use of HRME and Rx-DIS interactions in the outpatient elderly population. Using electronic communication allows pharmacists to provide meaningful interventions for numerous patients receiving care in a highvolume family medicine clinic setting.

J Manag Care Spec Pharm. 2017;23(5):520-24

Copyright $\odot 2017$, Academy of Managed Care Pharmacy. All rights reserved.

\section{What is already known about this subject}

Use of high-risk medications in the elderly has been associated with negative clinical outcomes such as confusion, falls, and mortality.

The 2015 Healthcare Effectiveness Data and Information Set (HEDIS) Measures list high-risk medication use and drug-disease interactions in the elderly as quality measures to be evaluated. Pharmacist involvement in geriatric care improves therapeutic, safety, hospitalization, and adherence outcomes.

\section{What this study adds}

Using clinical pharmacists to encourage the safe and effective use of medications in the outpatient elderly population reduces use of potentially inappropriate medications, thereby increasing compliance with HEDIS Measures.

Electronic communication is an efficient and effective method to reduce use of high-risk medications in the elderly population in a high-volume family medicine clinic setting.

$\mathrm{M}$ edication-related problems are common and costly, yet they are often preventable in older adults. It is estimated that $27 \%$ of adverse drug events in primary care and $42 \%$ in long-term care are preventable. ${ }^{1,2}$ In 2001, the total health care expenditures related to use of potentially inappropriate medications were estimated to cost the U.S. health care system up to $\$ 7.2$ billion. $^{3}$ In addition to the significant financial burden, medication-related problems in the older adult population have been linked to unfavorable clinical outcomes. The Beers Criteria for Potentially Inappropriate Medication Use in Older Adults, ${ }^{4}$ published by the American Geriatrics Society, provides an explicit list of potentially inappropriate medications that have been associated with unfavorable outcomes, such as confusion, falls, and mortality. The Beers Criteria is used widely in geriatric clinical care, education, research, and in the development of quality indicators. One of these quality indicators, the Healthcare Effectiveness Data and Information Set (HEDIS), is a performance measurement tool administered and coordinated by the National Committee for Quality Assurance.

The HEDIS Measures provide a retrospective review of services and performance of care for hospitals and clinics and is 
used by more than $90 \%$ of U.S. health plans. ${ }^{5}$ Results are used to measure performance, identify quality initiatives, provide educational programs for providers and members, ${ }^{5}$ and compare care between plans to assist in choosing which health plan to offer. Based on the Beers Criteria, the 2015 HEDIS Measures list use of high-risk medications in the elderly (HRME) and potentially harmful drug-disease (Rx-DIS) interactions in the elderly as quality measures to be evaluated. ${ }^{6-8}$ Additionally, HRME and Rx-DIS interactions have been significantly associated with mortality, hospital admission, and need for emergency care. ${ }^{9}$ One study suggests that pharmacist interventions to reduce use of HEDIS-defined HRME would improve the quality of care in older adults. ${ }^{8}$

Despite the increasing data on the clinical significance of HEDIS-defined HRME, no published studies to date have assessed the effect of pharmacist interventions on HRME and Rx-DIS interactions. Because the benefit of pharmacist involvement in outpatient geriatric care is well established, ${ }^{10}$ it is reasonable to hypothesize that pharmacists may assist in reducing use of HEDIS-defined HRME. Thus, the purpose of this study was to examine the effect of pharmacist intervention on HRME and Rx-DIS interactions in an outpatient elderly population.

\section{Methods}

\section{Study Design, Setting, and Patient Population}

This was an institutional review board-approved, retrospective cohort study conducted at a resident-based family medicine clinic. In this clinic, providers see an average of 125 patients daily, 35\% of whom are Medicare beneficiaries. In November 2014, a new service was implemented within the clinic. Using Centricity Practice Solutions 12, our electronic medical record (EMR), a residency-trained clinical pharmacist prospectively screened visits for HRME and Rx-DIS interactions in patients who were aged 65 years or older. Of note, the automated drug interaction software in Centricity Practice Solutions 12 requires prescribers to seek out the drug interaction information rather than presenting them with an interaction alert.

Patients were identified through the clinic schedule. If 2 or more HRME or at least 1 Rx-DIS interaction were noted, and these medications appeared to be of clinically significant risk according to the pharmacist's judgment, an alert was sent to the physician through the EMR before a patient's visit. This alert informed physicians of the pharmacist's findings, explained why the medication was potentially inappropriate, and if applicable, provided a recommendation for a safer alternative.

For the implemented intervention service, HRME referred to medications on the Beer's Criteria. ${ }^{7}$ Rx-DIS interactions for chronic kidney disease (CKD) referred to nonsteroidal anti-inflammatory drugs (NSAIDs). Rx-DIS interactions for a history of dementia consisted of antipsychotics, benzodiazepines, tricyclic antidepressants (TCAs), H2 receptor antagonists, nonbenzodiazepine hypnotics, and anticholinergics. Rx-DIS interactions for a history of falls consisted of anticonvulsants, nonbenzodiazepine hypnotics, selective serotonin receptor antagonists, antiemetics, antipsychotics, benzodiazepines, and TCAs. ${ }^{11}$

Patients were included in this study if they were aged at least 65 years, had at least 1 previous clinic visit, and were scheduled for an afternoon appointment. Only afternoon clinic visits were included because this was the time of day that pharmacists were available in the clinic to address any questions that physicians had in response to an electronic alert. Patients were excluded if they were seen in the clinic for a nonprimary care visit.

Patients who received the new pharmacist screening service between December 1, 2014, and April 8, 2015, were eligible for inclusion in the intervention group. The control group was composed of patients seen in the same clinic from March 1 to May 31, 2014. An "opportunity for intervention" was noted in the control group if 2 or more HRME and/or 1 or more Rx-DIS interaction was present and appeared to be of clinically significant risk (i.e., an electronic alert would have been sent had the patient been in the intervention group). The control patient's chart was then queried to determine if the noted HRME and/or Rx-DIS interaction was changed during the patient's clinic visit.

\section{Outcome Measurements}

The primary endpoint was the incidence of medication change in the intervention group as compared with the control group. Medication change was defined as discontinuation; switch to an alternative, safer agent; or reduced dosage or frequency. Secondary endpoints included the percentage of all patients with 2 or more HRME or with at least 1 Rx-DIS interaction, the most common HRME by class, percentage of changed medications added back at the end of the study period in the intervention group, the recommendation acceptance rate by resident physicians and faculty physicians, the most common medication classes changed, and the percentage of HRME or Rx-DIS interactions detected by the EMR interaction checker in the intervention group.

\section{Statistical Analysis}

Assuming a 5\% rate of change in the control group and a 30\% rate of change in the intervention group, 43 possible medication changes per group were needed to obtain a power of $80 \%$ and a two-sided alpha of 0.05 . The power calculation was empirically estimated by the study investigators because literature searches revealed no similarly designed studies on which to base the power calculation. The primary endpoint was analyzed via a chi square analysis. Secondary endpoints were analyzed with descriptive statistics, chi square, or Fisher's exact test. 


\section{Results}

\section{Baseline Characteristics}

During the study period, 143 patients in the intervention group met inclusion criteria. Of the 143 patients, 44 patients had at least $2 \mathrm{HRME}$ and/or at least $1 \mathrm{Rx}$-DIS interaction. An electronic alert was sent for 33 of the 44 patients.

In the control group, 115 patients met inclusion criteria. After a retrospective chart review, 31 patients had at least 2 HRME and/or 1 Rx-DIS interaction. Of the 31 patients, a clinically significant "opportunity for intervention" (OFI) was noted for 27 patients.

Baseline characteristics were similar between groups with the exception of age, sex, and the number of patients with dementia. The control group was significantly older, had a higher percentage of male patients, and had a lower percentage of patients with dementia as compared with the intervention group (Table 1).

\section{Primary Endpoint}

Thirty-three electronic alerts were sent to physicians in the intervention group, compared with 27 documented OFI in the control group. Of the patients who qualified for an electronic alert (intervention group) or OFI (control group), the median number of medication changes recommended per patient was 2 (interquartile range $=1$ ). Significantly more medication changes were made by physicians in the pharmacist intervention group as compared with physicians alone in the historical control group $(25.9 \%$ vs. $2 \%, P=0.001$, odds ratio $=17.1$, $95 \%$ confidence interval $=2.2-134.8$ ). Over $85 \%$ of the medication changes made in the intervention group were preserved at the end of the 17-week study period.

\section{Secondary Endpoints}

Sixty-two of the 258 total patients had at least 2 HRME (24\%), with 35 patients in the intervention group and 27 in the control group $(P=0.852)$. The most common medication classes included NSAIDs, benzodiazepines (short acting more common than long acting), anticholinergic agents, and skeletal muscle relaxants.

Of the total 258 patients, $13.6 \%$ had at least 1 Rx-DIS interaction, with 24 patients in the intervention group and 11 patients in the control group $(P=0.092)$. The number of patients with Rx-DIS interactions among subgroups with CKD, dementia, or a history of falls are listed in Table 2. There were significantly more patients with dementia and at least 1 Rx-DIS interaction in the intervention group as compared with the control group $(P=0.033)$.

The recommendation acceptance rate among faculty physicians was $21.7 \%$ as compared with $34.4 \%$ among resident physicians $(P=0.314)$. The most frequently changed medication classes were skeletal muscle relaxants, benzodiazepines, and NSAIDs.

\section{TABLE 1 Baseline Characteristics}

\begin{tabular}{l|rr|rr|r}
\hline & \multicolumn{2}{|c|}{$\begin{array}{c}\text { Intervention } \\
\mathbf{n}=\mathbf{1 4 3}\end{array}$} & \multicolumn{2}{|c|}{$\begin{array}{c}\text { Control } \\
\mathbf{n}=\mathbf{1 1 3}\end{array}$} & $\boldsymbol{P}$ Value \\
\hline Age, years (SD) & $72.1(6.1)$ & 74.4 & $(6.8)$ & 0.005 \\
\hline Sex, $\mathrm{n}(\%$ male) & $40(28.0)$ & $50(43.0)$ & 0.009 \\
\hline Race, $\mathrm{n}(\%$ Caucasian) & $128(89.5)$ & $94(81.7)$ & 0.070 \\
\hline Diabetes, $\mathrm{n}(\%)$ & $62(43.3)$ & $52(45.2)$ & 0.765 \\
\hline Hypertension, $\mathrm{n}(\%)$ & $123(86.0)$ & $96(83.5)$ & 0.572 \\
\hline Hyperlipidemia, $\mathrm{n}(\%)$ & $103(72.0)$ & $84(73.0)$ & 0.856 \\
\hline Coronary artery disease, $\mathrm{n}(\%)$ & $27(18.9)$ & $27(23.5)$ & 0.367 \\
\hline Congestive heart failure, $\mathrm{n}(\%)$ & $14(9.8)$ & $14(12.2)$ & 0.541 \\
\hline Chronic kidney disease, $\mathrm{n}(\%)$ & $29(20.3)$ & $32(27.8)$ & 0.156 \\
\hline Dementia, $\mathrm{n}(\%)$ & $15(10.5)$ & 4 & $(3.5)$ & 0.032 \\
\hline History of falls, $\mathrm{n}(\%)$ & $15(10.5)$ & 8 & $(7.0)$ & 0.322 \\
\hline SD standard deviation. & \multicolumn{4}{|l}{} \\
\hline
\end{tabular}

Of the 58 medication changes requested in the intervention group, 36 (62.1\%) were detected by the drug-interaction software. The majority of the alerts read, "Medication is potentially inappropriate or medication is absolutely contraindicated in patients aged 65 years or older." No Rx-DIS interactions were detected by the drug-interaction software.

\section{Discussion}

This study shows that physicians are significantly more likely to change high-risk medications in the outpatient elderly population when notified electronically of potential patient-specific medication problems by a pharmacist. Because HRME and Rx-DIS interactions have been linked to mortality, hospital admission, and need for emergency care, ${ }^{9}$ interventions such as these are prudent for not only the health and well-being of elderly patients, but also for reducing the financial burden on the health system.

Because this interventional study appears to be the first of its kind, direct comparison with other literature is limited. However, it is clear that this study validates current literature describing the benefit of pharmacist involvement in the care of geriatric patients. In 2013, Lee et al. performed a meta-analysis consisting of 20 studies that showed that pharmacist care is significantly superior to the comparison on therapeutic, safety, hospitalization, and adherence outcomes. ${ }^{10}$

The overall prevalence of Rx-DIS interactions in our study (13.6\%) is similar to previously published data (15.2\%). ${ }^{8}$ However, the prevalence of Rx-DIS interaction per disease state is much higher than quoted in previous literature (dementia $52.6 \%$ vs. $20.2 \%$, history of falls $82.6 \%$ vs. $16.2 \%$, and CKD $16.4 \%$ vs. $8.5 \%)^{8}$ This difference can be attributed to a much lower absolute number of patients with dementia, a history of falls, or CKD in our study in combination with the increased number of drug classes constituting Rx-DIS interactions since 2011. This theory explains the similar overall prevalence rate in 
TABLE 2 Prevalence of Patients with CKD, Dementia, a History of Falls, and 1 or More Rx-DIS Interaction

\begin{tabular}{|c|c|c|c|}
\hline & Intervention, $\mathbf{n}=29$ & Control, $n=32$ & $P$ Value \\
\hline CKD patients with $\geq 1$ Rx-DIS interaction, $\mathrm{n}(\%)$ & $6 \quad(20.7)$ & $4 \quad(12.4)$ & 0.496 \\
\hline \multirow[t]{2}{*}{ Most common CKD Rx-DIS interactions by class } & \multicolumn{3}{|c|}{ Nonaspirin NSAIDs } \\
\hline & Intervention, $\mathbf{n}=15$ & Control, $n=4$ & $P$ Value \\
\hline Dementia patients with $\geq 1$ Rx-DIS interaction, $\mathrm{n}(\%)$ & $10 \quad(66.6)$ & $0 \quad(0.0)$ & 0.033 \\
\hline \multirow[t]{2}{*}{ Most common dementia Rx-DIS interactions by class } & \multicolumn{3}{|c|}{ Benzodiazepines, atypical antipsychotics, nonbenzodiazepine hypnotics, and anticholinergics } \\
\hline & Intervention, $\mathbf{n}=15$ & Control, $n=8$ & $P$ Value \\
\hline Patients with a history of falls and $\geq 1$ Rx-DIS interaction, $\mathrm{n}(\%)$ & $12 \quad(80.0)$ & $7 \quad(87.5)$ & 1.00 \\
\hline Most common falls Rx-DIS interactions by class & \multicolumn{3}{|c|}{$\begin{array}{l}\text { SSRIs, benzodiazepines, atypical antipsychotics, anticonvulsants, nonbenzodiazepine } \\
\text { hypnotics, and TCAs }\end{array}$} \\
\hline
\end{tabular}

the setting of much higher individual prevalence rates for each disease state. Additionally, varying prescribing practices as a result of vastly different formulary systems in a civilian population compared with a Veterans Affairs population may have affected the observed rates, since previous literature on this topic has largely focused on the veteran population. Literature searches did not reveal published data on the prevalence of HRME in the outpatient elderly population, only incidence, which was not in the scope of this research.

Our study analyzed a novel intervention service that focused on implementing initiatives to reduce the use of highrisk medications in the older adult population. Some of the strengths of this service include involvement of residencytrained clinical pharmacists and interdisciplinary collaboration, as well as a focus on quality metric HEDIS Measures. Additionally, we were able to use electronic communication as an efficient and effective communication strategy. Strengths of this study include being the first published study to date that evaluates interventions to reduce use of HEDIS-defined HRME and Rx-DIS interactions by any health care professional. Additionally, this study also appears to be the first to examine HRME and Rx-DIS interactions in a civilian population, since many other studies have focused on veterans. ${ }^{8,9}$ Studying a civilian population has given us a more broad patient population to contribute to the body of literature on this topic.

\section{Limitations}

This retrospective observational study was small and single center in nature, thus introducing the potential for inherent bias and confounding. Additionally, the generalizability of the study may be limited to outpatient clinics that have an integrated clinical pharmacist as part of the medical team. Despite these limitations, the similarities to previously published literature with respect to the prevalence of Rx-DIS interactions increases the external validity of this study.

Only 4 patients in the control group (Table 1) had a diagnosis of dementia, which drastically reduced the chance of this group having a dementia Rx-DIS interaction compared with the intervention group. Our recommendation acceptance rates by resident and faculty physicians were lower than what is generally seen in the literature. ${ }^{12}$ However, no education was performed before study implementation in attempt to capture the providers' unbiased attention to high-risk medications. Additionally, physicians may not have seen electronic alerts before their visits with the patients, which likely biased the results in favor of the control group. Thus, if physicians had to acknowledge the alert when opening the chart, perhaps more recommendations would have been noted and accepted. Future initiatives would likely benefit from prescriber education before implementation. ${ }^{13}$

The intervention and control groups received care by the same physicians, with the exception of 1 residency class, thus introducing the possibility of prescribing differences among this group of 8 resident physicians. Also, it is important to note that this service relies on the EMR to aid in the pharmacist's judgment of clinical significance. Although not explored in our service, the pharmacist would ideally personally evaluate a patient before making a recommendation instead of solely assessing the patient's EMR. However, this was not possible based on the workflow of the clinic and the design of the new service.

Finally, the clinical accuracy and relevance of all drug-interaction software should be critically evaluated. Through this study, we found that just over $60 \%$ of all HRME and no Rx-DIS interactions were detected by Centricity Practice Solutions 12 . Additionally, the clinically significant interactions detected by the software seemed diluted with clinically insignificant interactions. Although other vendors and electronic systems may be more effective at detecting clinically significant interactions, evaluating and refining drug interaction software to aid in clinical decision making should be a primary modality to encourage the safe and effective use of medication, especially in the older population.

Future initiatives should investigate the efficacy and efficiency of different communication strategies. Additionally, identifying ways to efficiently target high-risk patients will be helpful in the development of future services. In our study, 
only about $30 \%$ of the patients screened in the intervention group were eligible for an electronic alert based on the criteria of 2 or more HRME or at least 1 Rx-DIS interaction. The significant time burden of reviewing charts of patients who are not eventually deemed eligible for the intervention may not be feasible for busy ambulatory settings. Thus, creation of a risk stratification tool or identifying patients based on use of specific high-risk medication classes could produce a higher yield for the invested time. These tools may also be valuable in capturing patients who have only 1 HRME.

\section{Conclusions}

$\overline{\text { Clinical pharmacy interventions resulted in significant reduc- }}$ tions in use of high-risk medications and drug-disease interactions in an outpatient elderly population. The most commonly changed medication classes were benzodiazepines, skeletal muscle relaxants, and NSAIDs. Electronic communication allowed pharmacists to provide meaningful interventions for numerous patients who were seen in a high-volume family medicine clinic setting. Using efficient methods for capturing high-risk patients will be prudent in future initiatives.

\section{Authors}

SARAH C. WEDDLE, PharmD, BCPS; A. SHAUN ROWE, PharmD, BCPS; and RACHEL C. RENWICK, PharmD, BCPS, University of Tennessee Health Science Center College of Pharmacy, Department of Clinical Pharmacy, and the University of Tennessee Medical Center, Department of Pharmacy, Knoxville. JULIE W. JETER, MD, University of Tennessee Graduate School of Medicine, Department of Family Medicine, Knoxville. SHAUNTA' M. CHAMBERLIN, PharmD, BCPS, FCCP, University of Tennessee Medical Center, Department of Pharmacy, and University of Tennessee Graduate School of Medicine, Department of Family Medicine, Knoxville. ANDREA S. FRANKS, PharmD, BCPS, University of Tennessee Health Science Center College of Pharmacy, Department of Clinical Pharmacy; University of Tennessee Medical Center, Department of Pharmacy; and University of Tennessee Graduate School of Medicine, Department of Family Medicine, Knoxville.

AUTHOR CORRESPONDENCE: Andrea S. Franks, PharmD, BCPS, UT College of Pharmacy, 1924 Alcoa Hwy., Box 117, Knoxville, TN 37920. Tel.: 865.974.2357; E-mail: afranks@uthsc.edu.

\section{DISCLOSURES}

There was no funding or sponsorship for this study. Rowe reports personal fees from The Medicines Company, outside the submitted work. The other authors have nothing to disclose.

Study concept and design were contributed by Jeter, Chamberlin, and Weddle, with assistance from Rowe and Franks. Weddle and Renwick collected the data, and data interpretation was performed by Weddle and Rowe, with assistance from Franks. The manuscript was written by Weddle and Rowe and revised by Weddle and Franks, assisted by Chamberlin.
The abstract for the completed study was presented at the American College of Clinical Pharmacy Global Conference, San Francisco, California, October 2015, and the Southeastern Residency Conference, Athens, Georgia, April 2015 (platform presentation). The research-in-progress abstract was presented at the Tennessee Society of Health System Pharmacists, Nashville, Tennessee, February 2015; the American Society of Health System Pharmacists Midyear Clinical Meeting, Anaheim, California, December 2014; and the University HealthSystem Consortium Pharmacy Council, Anaheim, California, December 2014

\section{ACKNOWLEDGMENTS}

The authors acknowledge Rebecca Higdon, MPH, who accessed and generated patient scheduling reports.

\section{REFERENCES}

1. Gurwitz JH, Field TS, Harrold LR, et al. Incidence and preventability of adverse drug events among older persons in the ambulatory setting. JAMA. 2003;289(9):1107-16.

2. Gurwitz JH, Field TS, Judge J, et al. The incidence of adverse drug events in two large academic long-term care facilities. Am J Med. 2005;118(3):251-58.

3. Fu AZ, Jiang JZ, Reeves JH, Fincham JE, Liu GG, Perri M 3rd. Potentially inappropriate medication use and healthcare expenditures in the US community dwelling elderly. Med Care. 2007;45(5):472-76.

4. American Geriatric Society 2015 Beers Criteria Update Expert Panel American Geriatrics Society 2015 Updated Beers Criteria for potentially inappropriate medication use in older adults. J Am Geriatr Soc. 2015;63(11): 2227-46

5. National Committee for Quality Assurance. HEDIS archives. HEDIS 2014 July 2014. Available at: http://www.ncqa.org/hedis-quality-measurement/ hedis-measures/hedis-archives. Accessed March 21, 2017.

6. Agency for Healthcare Research and Quality. Use of high-risk medications in the elderly: percentage of Medicare members 66 years of age and older who received at least two different high risk medications. October 2015. Available at: http://www.qualitymeasures. ahrq.gov/content. aspx?id=48658. Accessed March 21, 2017

7. Agency for Healthcare Research and Quality. Potentially harmful drugdisease interactions in the elderly: percentage of Medicare members 65 years of age and older who have evidence of underlying disease, condition, or health concern and who were dispensed an ambulatory prescription for a potentially harmful medication, concurrent with or after the diagnosis. October 2015. Available at: http://www.qualitymeasures.ahrq.gov/content. aspx?id=48654\&search=elderly. Accessed March 21, 2017.

8. Pugh MJ, Starner CI, Amuan ME, et al. Exposure to potentially harmful drug-disease interactions in older community dwelling veterans based on the Healthcare Effectiveness Data and Information Set Quality Measure: who is at risk? J Am Geriatr Soc. 2011;59(9):1673-78,

9. Pugh MJ, Marcum ZA, Copeland LA, et al. The quality of quality measures: HEDIS quality measures for medication management in the elderly and outcomes associated with new exposure. Drugs Aging. 2013;30(8):645-54.

10. Lee JK, Slack MK, Martin J, Ehrman C, Chisholm-Burns M. Geriatric patient care by U.S. pharmacists in healthcare teams: systematic review and meta-analyses. J Am Geriatr Soc. 2013;61(7):1119-27.

11. American Geriatric Society 2012 Beers Criteria Update Expert Panel. American Geriatric Society Updated Beers Criteria for potentially inappropriate medication use in older adults. J Am Geriatr Soc. 2012;60(4):616-31.

12. Mann A, Esse T, Abughosh SM, Serna O. Evaluating pharmacist-written recommendations to providers in a Medicare Advantage plan: factors associated with provider acceptance. J Manag Care Spec Pharm. 2016;22(1):49-55. Available at: http://www.jmcp.org/doi/10.18553/jmcp.2016.22.1.49.

13. Kaufman MB, Brodin KA, Sarafian A. Effect of prescriber education on the use of medications contraindicated in older adults in a managed Medicare population. J Manag Care Pharm. 2005;11(3):211-19. Available at: http://www.jmcp.org/doi/abs/10.18553/jmcp.2005.11.3.211. 